\title{
Tonsillar and Parotid Gland Metastases of Breast Cancer: Two Cases and A Literature Review
}

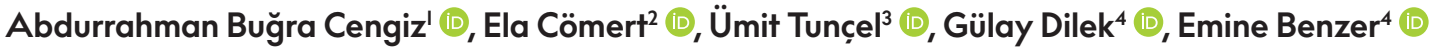 \\ 'Department of Otolaryngology, Bağcılar Training and Research Hospital, İstanbul, Turkey \\ ${ }^{2}$ Department of Otolaryngology, Kırıkkale University School of Medicine, Kırıkkale, Turkey \\ ${ }^{3}$ Department of Otolaryngology, Ankara Oncology Training and Research Hospital, Ankara, Turkey \\ ${ }^{4}$ Department of Pathology, Ankara Oncology Training and Research Hospital, Ankara, Turkey \\ ORCID IDs of the authors: A.B.C. 0000-0003-3942-6765; E.C. 0000-000I-7739-27I7; Ü.T. 0000-0002-3289-4075; G.D. 0000-0003-2458- \\ 578X; E.B. 0000-0002-1280-4204.
}

Cite this article as: Cengiz AB, Cömert E, Tunçel Ü, Dilek G, Benzer E. Tonsillar and Parotid Gland Metastases of Breast Cancer: Two Cases and A Literature Review. Cyprus J Med Sci 2019; 4(I): 57-9.

\begin{abstract}
Breast cancer is the most common tumor in women. One in eight women is affected by this disease in her lifetime. Both parotid gland and tonsillar metastases of breast cancer are extremely rare. Here, we present two separate cases of parotid gland and tonsillar metastases of breast carcinoma mimicking second primary tumors. The patient having parotid gland metastasis had disseminated disease and received palliative chemotherapy and hormone therapy. The other patient who had tonsillar metastasis underwent surgery without significant morbidity. Surgical resection of isolated tonsillar metastasis appears to have an influence on survival as the patient is free of disease at 12 months post-surgery.
\end{abstract}

Keywords: Breast neoplasms, neoplasm metastasis, palatine tonsil, parotid gland

\section{INTRODUCTION}

Breast cancer is one of the most common neoplasms in women. The most common sites of metastasis are the bone, lungs, liver, lymph nodes, and brain. Metastasis to the head and neck region, such as tonsil and parotid gland, is uncommon. As the parotid gland includes the lymph nodes inside the gland, metastasis to the parotid gland from the head and neck region is not rare; however, parotid gland metastasis of malignant tumors of infraclavicular origin is uncommon. Unlikely, tumor metastasis to the tonsil is extremely rare, accounting for only $0.8 \%$ of all tonsil tumors (I).

Herein, we present two separate cases of parotid gland and tonsillar metastases of breast carcinoma mimicking second primary tumors and a literature review.

\section{CASE PRESENTATIONS}

\section{Case I}

A 54-year-old woman was presented to our department with a complaint of progressive painless swelling in the left parotid gland since the previous 3 months. Upon examination, a solitary swelling of approximately $3 \times 2 \mathrm{~cm}$ was observed on the left parotid region. Fistulization of the mass to the skin was observed, and the overlying skin was hyperemic. Facial nerve functions were normal. She had a history of grade 3 invasive ductal carcinoma of left breast with multiple bone metastases at the vertebrae, costas, right iliac bone, and cranium. She underwent surgery, radiotherapy, and chemotherapy for breast carcinoma.

Computed tomography (CT) and ultrasound examination of the parotid region revealed a $3 \times 2.5 \mathrm{~cm}$ mass in the left parotid gland. Pathological examination of fine needle aspiration biopsy revealed a malignant epithelial tumor. Both primary parotid malignancy and metastasis from breast cancer were considered in differential diagnosis. An incisional biopsy was performed, and pathological examination revealed a poorly differentiated estrogen receptor-positive adenocarcinoma 
and a progesterone receptor-negative adenocarcinoma with focal cytoplasmic staining with mammaglobin, supporting the possibility of parotid gland metastasis from breast carcinoma (Figure I). The test for c-erbB-2 was negative according to immunohistochemical examination. The patient was treated with chemotherapy and hormone therapy after diagnosis. She was alive with disease 8 months after the diagnosis of parotid metastasis.

\section{Case 2}

A 60-year-old woman presented to our department with sore throat and swelling of her left tonsil since the previous 2 months. She had a history of grade 2 invasive ductal carcinoma of right breast treated using modified radical mastectomy, postoperative radiotherapy, and chemotherapy 2 years ago. Physical examination showed a unilateral enlargement of the left tonsil. CT of the oropharynx and the neck revealed a $5 \times 3-\mathrm{cm}$ mass in the left tonsillar region. Incisional biopsy was performed, and pathological examination revealed poorly differentiated car-

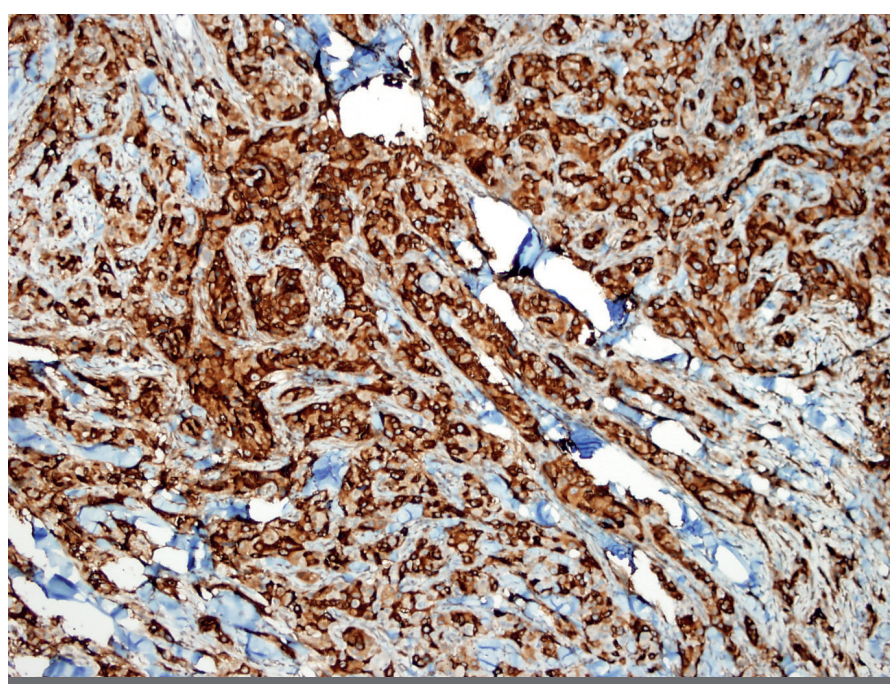

FIGURE I. Photomicrograph of a section through the parotid tumor revealing a metastatic invasive lobular carcinoma of the breast with focal cytoplasmic staining with mammaglobin ( $\times 400$ magnification)

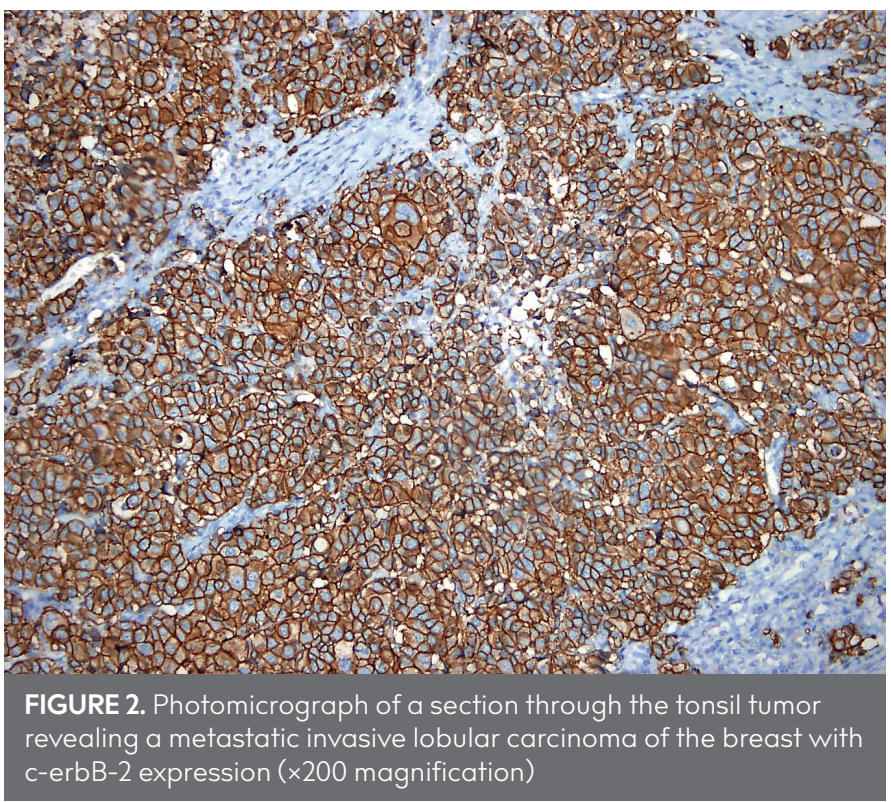

cinoma. Positron-emission tomography/CT demonstrated an increased uptake (SUV $V_{\text {max }}=29$ ) at the left tonsil and left deep cervical lymph nodes that indicated a second primary tonsillar carcinoma. There was no evidence of distant metastasis.

Based on these results, the tumor was considered a second primary tonsillar carcinoma. Intraoral resection of the tumor combined with left functional neck dissection was performed. Pathological examination of the specimen revealed a ductal carcinoma infiltration of the tonsil according to diffuse cytoplasmic staining with CK7 and focal cytoplasmic staining with mammaglobin. Some positive reactions were observed with c-erbB-2 and gross cystic disease fluid protein I5 (GCDFP-I5); however, no staining was observed with CK20 (Figure 2). In immunohistochemical examination, tumor was negative for estrogen and progesterone receptors and positive (score 3) for HER2. Similar histological findings were observed in eight lymph nodes. The patient received hormone therapy post-surgery and was free of disease at 12 months post-surgery. Written informed consent was obtained from both patients who participated in the study.

\section{DISCUSSION}

Breast cancer is the most common tumor in women. One in eight women is affected by this disease in her lifetime. The median survival of metastatic breast cancer is 12 to 24 months (2). Tumor stage and lymph node involvement are the most accurate prognostic indicators for a patient with breast cancer. Metastasis of breast carcinoma is rarely observed in the head and neck region; the most common areas are the brain and skull bones.

Metastasis from distant tumors accounts for $<1 \%$ of all malignancies of the oral, maxillofacial, and upper neck regions (3). Metastatic tumors of the head and neck region usually originate from the lungs, breast, kidneys, thyroid gland, and prostate gland (3).

Metastatic involvement of the parotid gland is often secondary to squamous cell carcinoma of the head and neck and melanomas of the neighboring skin. Additionally, primary tumors other than the head and neck region, such as breast, prostate, kidneys, and gastrointestinal tumors, have the capability to metastasize to the parotid gland through the thoracic duct or the Batson's paraspinal venous plexus, bypassing the pulmonary vascular filtration (4). The treatment options for metastatic disease of the parotid gland include parotidectomy, radiation therapy, chemotherapy, and hormone therapy (5). Furthermore, all the current therapeutic procedures for metastatic disease of the parotid gland are palliative in advanced disease.

The status of estrogen and progesterone receptors can vary between primary sites to metastasis for breast carcinoma. This suggests a dedifferentiation of the tumor (6). Therefore, hormone receptor analysis of the metastasis is warranted by surgical biopsy. HER2 testing of metastasis does not appear to have any influence on treatment decisions (6).

Perez et al. (7) reported a patient with parotid gland metastasis that occurred 5 years after the treatment of breast carcinoma. They suggested that the immunohistochemical study of estrogen receptor is fundamental for diagnosis of parotid gland metastasis. Dangore et al. (8) reported a patient with swelling in the 
left parotid gland, who was previously diagnosed with breast cancer. Based on the clinical findings, second primary malignancy of the parotid gland was suspected, and parotidectomy was performed (8). Additionally, the patient received chemoradiotherapy. They suggested that swelling in the parotid gland needed to be examined very carefully. Ando et al. (5) reported a patient with advanced breast cancer and swelling in the parotid gland. They also performed parotidectomy for diagnosis. Their patient received capecitabine in addition to trastuzumab postoperatively, which is one of the treatments used for patients with HER2-positive breast cancer.

In the literature, to the best of our knowledge, 9 out of 17 patients presenting with parotid gland metastasis underwent parotidectomy. Most of the cases were performed due to suspicion of second primary tumor. In the case of disseminated metastasis of breast cancer, resection of parotid metastasis does not improve survival rates (5). In the case of isolated parotid gland metastasis, parotidectomy could be the preferred choice of treatment, and it may indicate the percentage of hormone receptors requiring adjuvant hormone therapy.

Metastatic tumors involving the tonsils are rare. In the literature, only a few cases of tonsillar metastasis as a presenting feature have been reported (9). Metastasis to the tonsil is more often reported unilaterally with a preference for the left side, although primary tumor is in the right breast (9). In general practice, late-onset metastasis has better prognosis than early metastasis in breast cancer. Patients with tonsillar metastasis of breast cancer have poor prognosis regardless of the location of the primary tumor, its histological type, and the treatment modality (9).

Tonsillar metastasis is often associated with multifocal disseminated disease. However, the patient in our report with tonsillar metastasis had no evidence of recurrent tumor or other distant metastasis. Pathological evaluation of preoperative tonsillar biopsy did not help us distinguish between primary and metastatic tumor of the tonsil. The tumor was considered to be a second primary tonsillar carcinoma, and surgical treatment was performed with curative intent. Breast was confirmed as the origin of metastasis by the presence of GCDP-I5 in the postoperative pathological examination of the tonsil and lymph nodes of the neck.

Patients with tumors that overexpress HER2 protein are candidates for therapies that significantly reduce mortality (I0). In our case study, immunohistochemical analysis was negative for estrogen and progesterone receptors and positive for HER2. Therefore, trastuzumab therapy was continued postoperatively. The presence of disseminated disease is probably the main factor in determining prognosis. Surgical resection of tonsillar metastasis appears to have an influence on survival because our Case 2 patient is free of disease at 12 months post-surgery.

Informed Consent: Written informed consent was obtained from the patients who participated in this study.

Peer-review: Externally peer-reviewed.

Author contributions: Concept - A.B.C., E.C., G.D., E.B.; Design - A.B.C., E.C., G.D., E.B.; Supervision - E.C., Ü.T.; Resource - A.B.C., E.C., G.D., E.B.; Materials - G.D., E.B.; Data Collection and/or Processing - A.B.C., E.C., G.D., E.B.; Analysis and/or Interpretation - A.B.C., E.C., G.D., E.B.; Literature Search - E.C., Ü.T.; Writing - A.B.C., Ü.T.; Critical Reviews - E.C.

Conflict of Interest: The authors have no conflicts of interest to declare.

Financial Disclosure: The authors declared that this study has received no financial support.

\section{REFERENCES}

I. Luini A, Gatti G, Diaz J, Botteri E, Oliveira E, Cecilio Sahium de Almeida $\mathrm{R}$, et al. Angiosarcoma of the breast: the experience of the European Institute of Oncology and a review of the literature. Breast Cancer Res Treat 2007; 105: 81-5. [CrossRef]

2. Jemal A, Bray F, Center MM, Ferlay J, Ward E, Forman D. Global cancer statistics. CA Cancer J Clin 2011; 61: 69-90. [CrossRef]

3. Al-Benna S, Tzakas E. Submental lymph node metastasis from invasive ductal breast carcinoma. Arch Gynecol Obstet 2012; 285: II53-6. [CrossRef]

4. Nuyens M, Schüpbach J, Stauffer E, Zbären P. Metastatic disease to the parotid gland. Otolaryngol Head Neck Surg 2006; 135: 844-8. [CrossRef]

5. Ando K, Masumoto N, Sakamoto M, Teraoka K, Suzuki T, Kurihara T, et al. Parotid Gland Metastasis of Breast Cancer: Case Report and Review of the Literature. Breast Care (Basel) 20Il; 6: 47I-3. [CrossRef]

6. Sproson EL, Herd MK, Spedding AV, Brennan PA, Puxeddu R. Treatment of breast adenocarcinoma metastasis to the neck: dedifferentiation of the tumor as suggested by hormone markers. Head Neck 2012; 34: 1095-9. [CrossRef]

7. Perez-Fidalgo JA, Chirivella I, Laforga J, Colio JM, Blanes MD, Baydal R, et al. Parotid gland metastasis of a breast cancer. Clin Transl Oncol 2007; 9: 264-5. [CrossRef]

8. Dangore-Khasbage SB, Degwekar SS, Bhowate RR, Bhake A. Metastatic involvement of parotid from carcinoma of the breast--a case report. Oral Maxillofac Surg 2009; 13: 49-53. [CrossRef]

9. Bar R, Netzer A, Ostrovsky D, Daitzchman M, Golz A. Abrupt tonsillar hemorrhage from a metastatic hemangiosarcoma of the breast: case report and literature review. Ear Nose Throat J 20ll; 90: Il6-20. [CrossRef]

10. Gutierrez C, Schiff R. HER2: biology, detection, and clinical implications. Arch Pathol Lab Med 20II; 135: 55-62. 